International Journal of Advanced Chemistry, $8(2)(2020) 209-216$
International Journal of Advanced Chemistry
WPC
Website: www.sciencepubco.com/index.php/IJAC
Research paper

\title{
Molecular interaction on imidazolium based ionic liquids and serum albumin: A spectroscopy approach
}

\author{
Manoj Kumar Banjare ${ }^{1,2 *}$, Ramesh Kumar Banjare ${ }^{2}$, Kallol Kumar Ghosh ${ }^{1 *}$ \\ ${ }^{1}$ School of Studies in Chemistry, Pt. Ravishankar Shukla University, Raipur (C.G.) 492010, India \\ ${ }^{2}$ MATS School of Sciences, MATS University, Pagaria Complex, Pandri, Raipur, C.G. 492013, India \\ *Corresponding authorE-mail: manojbanjare7@gmail.com, kallolkghosh@gmail.com
}

\begin{abstract}
Interaction between globular proteins with imidazolium based ionic liquids (ILs) is extremely significant considering that of the vast use of ILs, since protein stabilizer in the current years. The present work, the interaction of human serum albumin (HSA) and bovine serum albumin (BSA) with 1-butyl-3-methylimidazolium octylsulphate (BmimOS) and 1-decyl-3-methylimidazolium tetrafluoroborate $\left(\mathrm{DmimBF}_{4}\right)$ have been examine the use of fluorescence and FTIR. Fluorescence spectra of HSA/BSA are extinguished by BmimOS $/ \mathrm{DmimBF}_{4}$ ILs by way of the dynamic method. The various thermodynamic parameters were revealing that very susceptible interactions exist between HSA/BSA and BmimOS /DmimBF 4 . The conformational adjustments of HSA/BSA were observed by means of FTIR analysis. Fluorescence methods were completed to find out about the thermal balance of HSA/BSA at different temperature. The thermal balance of BSA in the presence of ILs follows the style BmimOS/DmimBF 4 and presence of extra hydrophobic IL, decay increases swiftly as a characteristic of concentration.
\end{abstract}

Keywords: FTIR; Fluorescence; Ionic Liquids; Serum Albumin; Complexation.

\section{Graphical abstract}

Interaction of human serum albumin (HSA) and bovine serum albumin (BSA) with 1-butyl-3-methylimidazolium octylsulphate (BmimOS) and 1-decyl-3-methylimidazolium tetrafluoroborate $\left(\right.$ DmimBF$\left._{4}\right)$ have been study by fluorescence and FTIR. Fluorescence of HSA/BSA is quenched by $(\mathrm{BmimOS}) /\left(\mathrm{DmimBF}_{4}\right) \mathrm{ILs}$ with the steady state fluorescence method.

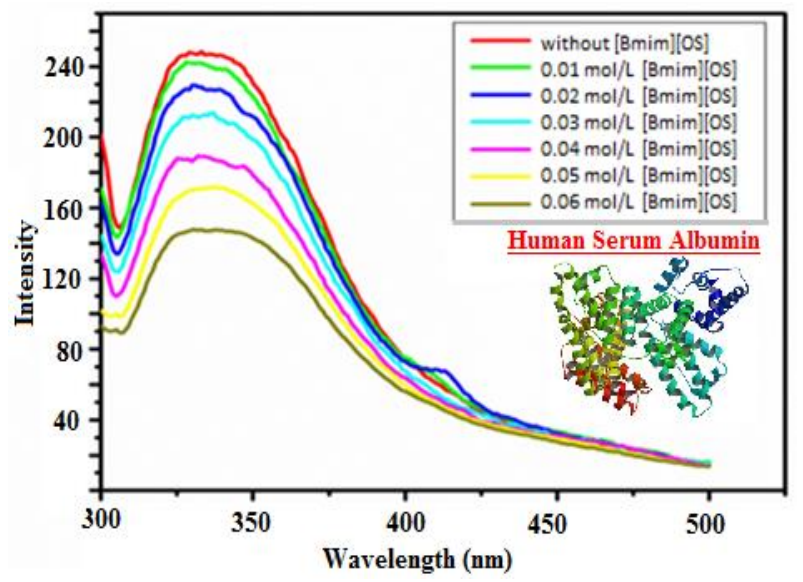

\section{Introduction}

Effect of proteins on the ionic liquids (ILs) provides important information about interactions between them which can change the secondary or tertiary structure of protein or denature them, thereby modifying the function of the molecule (Venkatesu et al. 2012, Venkatesu et al. 2014, Weingartner et al. 2012, Zhao et al. 2005). When a protein is denatured, the characteristic 3D arrangement is disorder (the protein unfolds) and as a result, its biological function is lost. Describing is an abrupt method that receive place within a partial variety of environment (Steinhauser et al. 2011). The abruptness of the unfolding transition indicates a cooperative process (Garab et al. 
2001). Protein-ILs systems have manifold applications in food industry, pharmaceutical industry and analytical biochemistry (Crevenna et al. 2012, Coutinho et al. 2012, Figueiredo et al. 2013, Shao et al. 2013, Perssion et al. 2012).

ILs are the combination of two cation and anion, have emerged as the capable solvents that have captured special attention from biotechnology and biophysical chemistry (Pal et al. 2015, Chai et al. 2015, Cummings et al. 2015). ILs represents an exciting novel class of cosolvents for biochemical process that include protein stability. The present study is contributed to improve considerate of function of the molecular structure of ILs in protein-ILs interactions and as a result, in the behaviour of ionic ILs since denaturants (Schroder et al. 2016, Sairi et al. 2014, Roy et al. 2016).

Kumar et al. 2012, studied the structural modify of bovine serum albumin (BSA) and 3-methyl-1-octylimidazolium chloride and 1-butyl3-methylimidazolium octylsulfate have been studied by spectroscopic methods. The interactional behaviour of ILs toward BSA in diverse ILs concentration systems at the air/solution interface and the bulk is study. CD mutual with the fluorescence provided expensive nearby into the unfolding of BSA as a result of IL binding. Patel et al. 2016, studied the interaction among N, N-dimethyl-2oxopyrrolidinium iodide and BSA by fluorescence, UV-vis and FTIR in mixture among molecular docking method. The FTIR results confirm the conformational modify of BSA on binding with N, N-dimethyl-2-oxopyrrolidinium. Patel et al. 2017, studied the interaction between N-butyl-N-methyl-morpholinium bromide, [Mor1,4] [Br] IL with human serum album (HSA). The result was analyzed by CD spectroscopic, UV-vis, fluorescence, and molecular docking method. The results show that $[\mathrm{Mor} 1,4][\mathrm{Br}]$ has a binding site on HSA and bind in the hydrophobic pocket of sub domain IIA of HSA. It was observed that [Mor1,4] [Br]IL maintain native conformation of HSA positive to definite concentration variety.

In our earlier work (Ghosh et al. 2018, Ghosh et al. 2016), we successfully deal with the interaction between globular protein with carbon quantum dot and surfactants. Herein, the present investigated the molecular interaction between ionic liquids i.e., 1-butyl-3-methyl imidazolium octyl sulfate (BmimOS) and 1-decyl-3-methylimidazolium tetrafluoroborate $\left(\mathrm{DmimBF}_{4}\right)$ and serum albumins i.e., human serum albumin (HSA), bovine serum albumin (BSA) by fluorescence and FTIR spectroscopy. Gibbs free energy changes of ILs and serum albumin has also been calculated. The present works give precious information concerning the mechanism of interaction of ILs and serum albumins. Furthermore, the current research supply as a important for improved considerate of the vital behaviour of ILs in industrial application. Molecular structures of ionic liquids, human serum albumin and bovine serum albumin are shown in Scheme1.

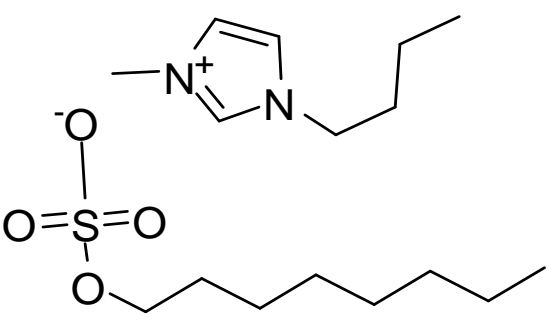

1-Butyl-3-methylimidazolium octylsulphate

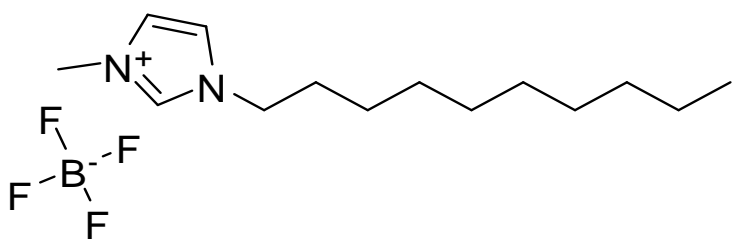

1-Decyl-3-methylimidazolium tetrafluoroborate

Scheme 1: Molecular Structure of Ionic Liquid 1-Butyl-3-Methylimidazolium Octylsulphate and 1-Decyl-3methylimidazolium Tetrafluoroborate.

\section{Experimental and methods}

\subsection{Materials}

1-Butyl-3-methylimidazolium octylsulphate, 1-decyl-3methylimidazolium tetrafluoroborate, human serum albumin and bovine serum albumin, potassium bromide was obtained sigma Aldrich Pvt. Ltd.

\subsection{Fluorescence spectrometry}

Fluorescence spectra of two BSA/HSA with changed concentration of ILs i.e., 1-Butyl-3-Methylimidazolium Octylsulphate (BmimOS) and 1Decyl-3methylimidazolium Tetrafluoroborate $\left(\mathrm{DmimBF}_{4}\right)$ were recorded at $295 \mathrm{~K}, 298 \mathrm{~K}$ and $305 \mathrm{~K}$ temperature in the wavelength range between 200-500 nm.

\subsection{FT-IR spectrometry}

FT-IR spectra were performed on a Nicolet iS10 FTIR (Thermo Fisher) by KBr pellets. The FTIR data of HSA and BSA during the existence and deficiency of ILs at $298 \mathrm{~K}$ were study in the range of $4000-400 \mathrm{~cm}^{-1}$. The FT-IR spectra of serum albumins and HSA-ILs, BSA-ILs structure are evidence during the range of $4000-400 \mathrm{~cm}^{-1}$.

\section{4. pH meter}

pH learn was prepared with a crucial $\mathrm{pH}$ meter (pH 700 EUTECH instrument).

\section{Results and discussion}

\subsection{Impact of ionic liquids on BSA/HSA}

Basic fluorescence of globular albumins is mostly endorsed in single-handed tryptophan residue ${ }^{23}$ and fluorescence quenching efficiency of albumins by other molecules was broadly studied by fluorescence quenching method (Xiao et al. 2016, Kanvah et al. 2019). The present investigation in molecular interaction between ILs [BmimOS]/ [DmimBF $]$ and HSA and BSA, the influence of ILs at different concentration by using steady-state fluorescence and observed result are shown in Fig. 1. Serum albumin was showed the tough fluorescence emission under excitation wavelength of $\lambda_{\max }=340 \mathrm{~nm}$ (Ghosh et al. 2018, Ghosh et al. 2016). Furthermore, [BmimOS]/ [DmimBF 4 
emitted nearly no fluorescence spectra below the same surroundings, which suggested that ILs might not affect the fluorescence belongings of serum albumins on all. Provisional, the basic fluorescence intensity of serum albumins was quenched regularly through the raise of the attentiveness of ILs which shows that ILs quenched the intrinsic fluorescence of albumins mainly during concentration dependent relative behaviour. The current result approved with the Stern-Volmer early linearity at higher concentrations of ILs.

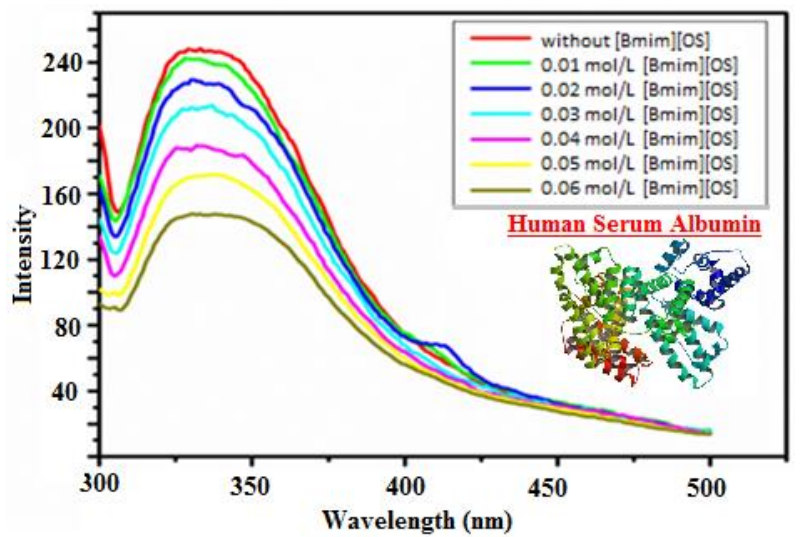

Fig. 1: Fluorescence Spectra of Human Serum Albumin into the Incidence of Different Concentration of 1-Butyl-3-Methylimidazolium Octylsulphate [Bmimos].

Steady static quenching mechanism is generally order like two types; (i) quenching and (ii) dynamic (Arik et al. 2011, Turner et al. 2019). Steady static fluorescence method reduce by the raise of temperature used for static quenching, since rising the temperature resulted rise in the flow coefficient. As a result, the persuade of different concentration of ILs on the fluorescence intensity of serum albumins on $298 \mathrm{~K}$ are shown in Figure.1. Fluorescence static quenching constants of BSA-BmimOS, HSA-BmimOS and BSA-DmimBF 4 , HSA$\mathrm{DmimBF}_{4}$ system at 3 different temperatures can be considered using Stern-Volmer equation (1) (Ghosh et al. 2018, Yi and Liu et al. 2019).

$\ln \frac{\mathrm{I}_{0}}{\mathrm{I}}=1+\mathrm{K}_{\mathrm{sv}}[\mathrm{Q}]$

Herein $\mathrm{I}_{0}$ and $\mathrm{I}$ are the intensities of fluorescence within the occurrence and deficiency of ILs, correspondingly. Hence, the Ksv is considered by using the linear decay of the plot of $\mathrm{I}_{0} / \mathrm{I}$ vs [Q] according to the equation (1). The graph plots on Stern-Volmer plots of BSABmimOS, HSA-BmimOS and BSA-DmimBF 4 system at two changed temperatures are revealed in Fig 2 and the values were scheduled here Table 1. As a results the Stern-Volmer quenching constant $\mathrm{K}_{\mathrm{Sv}}$ are decreasing according with increased temperature. This is confirm that the steady static quenching mechanism of BSA-BmimOS, HSA-BmimOS and BSA-DmimBF 4 , HSA-DmimBF 4.

\subsection{Calculate the binding constant and binding number}

The fluorescence static quenching method (Behere et al. 2019) to calculate the binding constant ( $\left.\mathrm{K}_{\mathrm{b}}\right)$ as well as binding amount (n) was calculated using subsequent equation (2).

$\log \frac{\left(\mathrm{I}_{0}-\mathrm{I}\right)}{\mathrm{I}}=\log \mathrm{K}_{\mathrm{b}}+\mathrm{n} \log [\mathrm{Q}]$

Graph plot between $\log \left(\mathrm{I}_{0}-\mathrm{I}\right) / \mathrm{I}$ vs $\log [\mathrm{Q}]$, as a results a straight line observed and the number of binding sites (n) and binding constant $\left(\mathrm{K}_{\mathrm{b}}\right)$ was achieved is shown in figure 3-4. In Table 1, shows that the value of binding constant $\left(\mathrm{K}_{\mathrm{b}}\right)$ increase significantly with the increase of temperatures, representing that the binding ability of ILs-BSA/HSA systems would be greatly increased when the temperature increased. These phenomenons have shown that ILs bound stoutly with BSA/HSA according to mole ratio of 1:1 and self-sufficient of the binding of ILs to BSA/HSA. Tayyab et al. ${ }^{30}$ studied the interaction of bovine serum albumin and bilirum (BR) by absorption spectroscopy. Analysis the association constant $(\mathrm{K})$ of BSA and BR is $6.9 \times 104 \mathrm{M}^{-1}$. As a result suggest that ErB binds to a site in the vicinity of BR binding site on BSA (Seetharamappa et al. 2004).
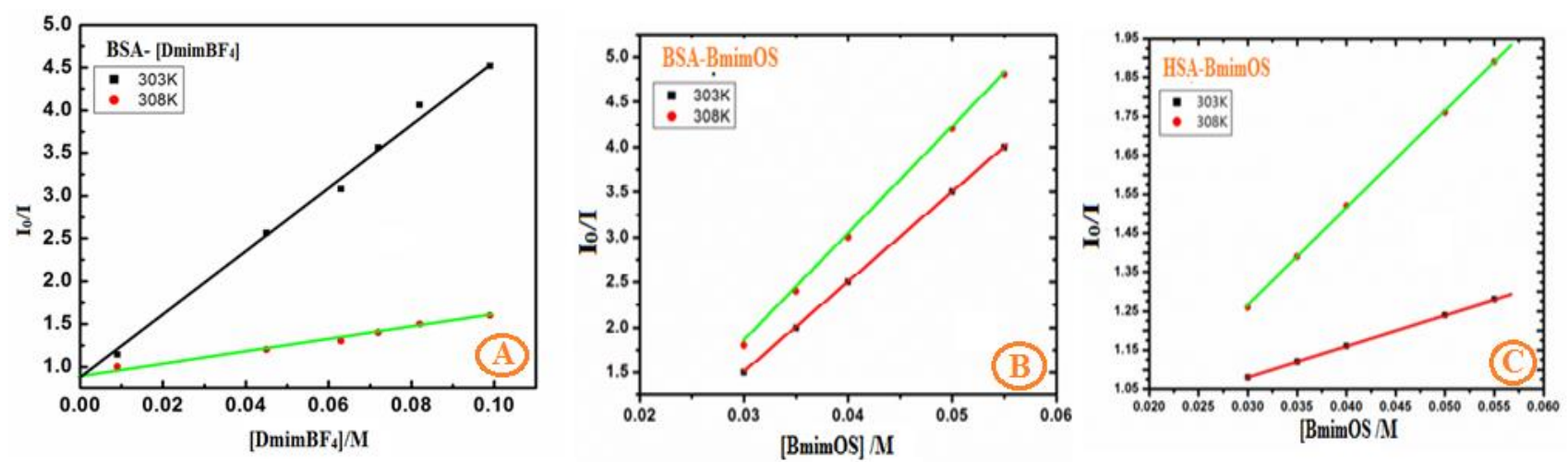

Fig. 2: [A] Graph Plot Between $\mathrm{I}_{0} / \mathrm{I}$ Vs Concentration of [Dmimbf $\left.{ }_{4}\right](\mathrm{M})$. [B] Graph Plot Between $\mathrm{I}_{0} / \mathrm{I}$ Against Concentration of [Bmimos] (M). [C] Graph Plot Between $\mathrm{I}_{0} / \mathrm{I}$ vs. Concentration of $[\mathrm{Bmimos}](\mathrm{M})$. 


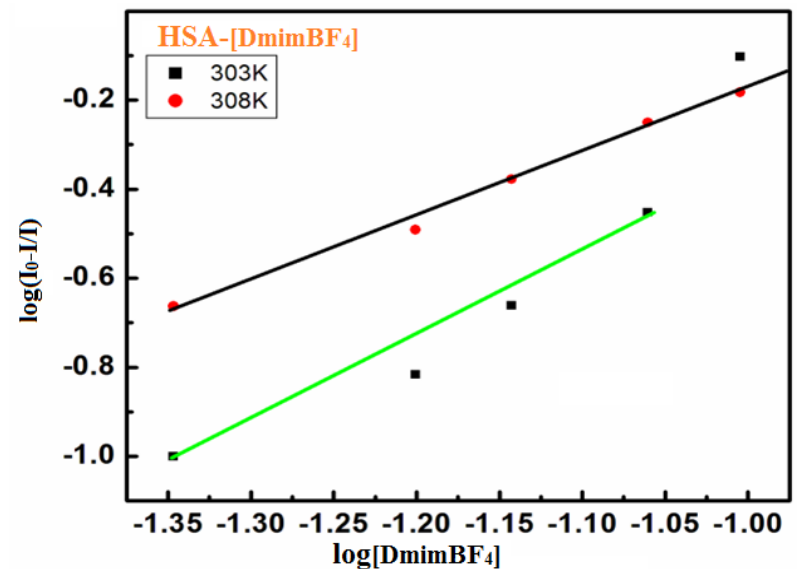

Fig. 3: Graph Plot between $\log \left[\left(\mathrm{I}_{0}-\mathrm{I}\right) / \mathrm{I}\right]$ Against $\log \left[\mathrm{Dmimbf}_{4}\right]$ for Binding Constant of HSA

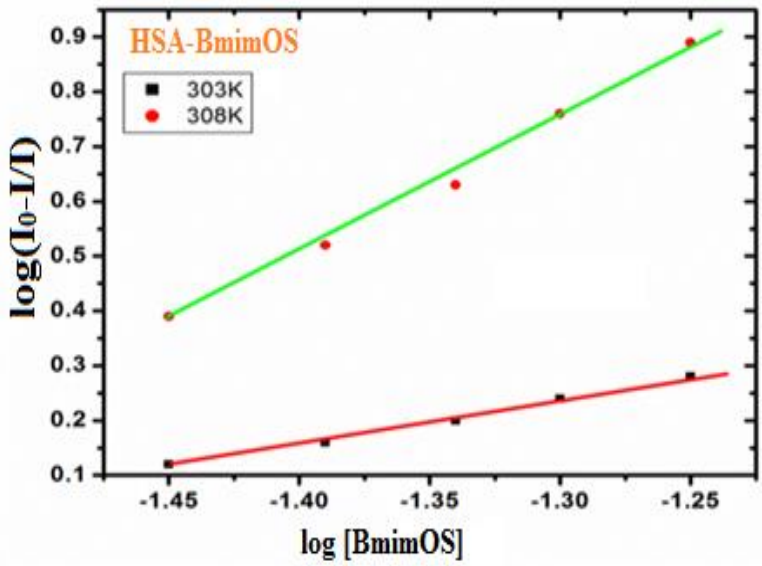

Fig. 4: Graph Plot between $\log \left[\left(\mathrm{I}_{0}-\mathrm{I}\right) / \mathrm{I}\right]$ Against Log $[\mathrm{Bmimos}]$ for Binding Constant Of HSA.

The Gibbs free energy change $(\Delta \mathrm{G})$ for the complexation can be considered from the binding constant value (Ka) using the following equation (3).

$\Delta \mathrm{G}=-2.303 \mathrm{RT} \log \mathrm{Ka}$

Where, $\Delta \mathrm{G}$ is the standard Gibbs free energy, $\mathrm{R}$ is gas constants $(8.314 \mathrm{~J} / \mathrm{mol} \mathrm{K}$ ), $\mathrm{T}$ is temperature (in Kalvin), Ka is binding constants. The calculated values of $\Delta \mathrm{G}$, the complexation process are found to be presented here Table 2 . The negative values of $\Delta \mathrm{G}$ indicate the spontaneity of complexation method at 298/303/308K temperature. In general, the binding constants/ interaction between BSA/HSA and ILs may electrostatic interaction, H-bonding, hydrophobic force and van der Waal interaction. Binding military can be determined by variant some thermodynamic parameters i.e., enthalpy change $(\Delta \mathrm{H})$, entropy change $(\Delta \mathrm{S})$ and Gibbs free energy change $(\Delta \mathrm{G})(\mathrm{Ghosh}$ et al. 2018).

Table 1: Binding Constant $\left(\mathrm{K}_{\mathrm{a}}\right)(\mathrm{Mol} / \mathrm{L})$ of $[\mathrm{Bmim}][\mathrm{OS}] /[\mathrm{Dmim}]\left[\mathrm{BF}_{4}\right]$ with Serum Albumins

\begin{tabular}{|c|c|c|c|c|c|c|c|c|}
\hline \multirow[t]{2}{*}{ Temperature (Kelvin) } & \multicolumn{4}{|c|}{ [BmimOS] } & \multicolumn{4}{|c|}{$\left[\mathrm{DmimBF}_{4}\right]$} \\
\hline & HSA & $\mathrm{R}$ & BSA & $\mathrm{R}$ & HSA & $\mathrm{R}$ & BSA & $\mathrm{R}$ \\
\hline 298 & 20.50 & 0.996 & 20.02 & 0.979 & 23.65 & 0.980 & 14.32 & 0.966 \\
\hline 303 & 28.43 & 0.991 & 36.98 & 0.993 & 36.0 & 0.957 & 28.0 & 0.992 \\
\hline 308 & 49.02 & 0.998 & 61.36 & 0.995 & 39.34 & 0.993 & 16.79 & 0.991 \\
\hline
\end{tabular}

\subsection{Binding interaction between ionic liquids and serum albumins}

The Gibbs free energy change $(\Delta \mathrm{G})$ for the complexation can be considered from the binding constant value (Ka) using the following equation (3).

\section{$\Delta \mathrm{G}=-2.303 \mathrm{RT} \log \mathrm{Ka}$}

Where, $\Delta \mathrm{G}$ is the standard Gibbs free energy, $\mathrm{R}$ is gas constants $(8.314 \mathrm{~J} / \mathrm{mol} \mathrm{K}), \mathrm{T}$ is temperature (in Kalvin), Ka is binding constants. The calculated values of $\Delta \mathrm{G}$, the complexation process are found to be presented here Table 2 . The negative values of $\Delta \mathrm{G}$ indicate the spontaneity of complexation method at 298/303/308K temperature. In general, the binding constants/ interaction between BSA/HSA and ILs may electrostatic interaction, H-bonding, hydrophobic force and van der Waal interaction. Binding military can be determined by variant some thermodynamic parameters i.e., enthalpy change $(\Delta \mathrm{H})$, entropy change $(\Delta \mathrm{S})$ and Gibbs free energy change $(\Delta \mathrm{G})(\mathrm{Ghosh}$ et al. 2018).

$\operatorname{InK}_{\mathrm{a}}=-\frac{\Delta \mathrm{H}}{\mathrm{RT}}+\frac{\Delta \mathrm{S}}{\mathrm{R}}$ 

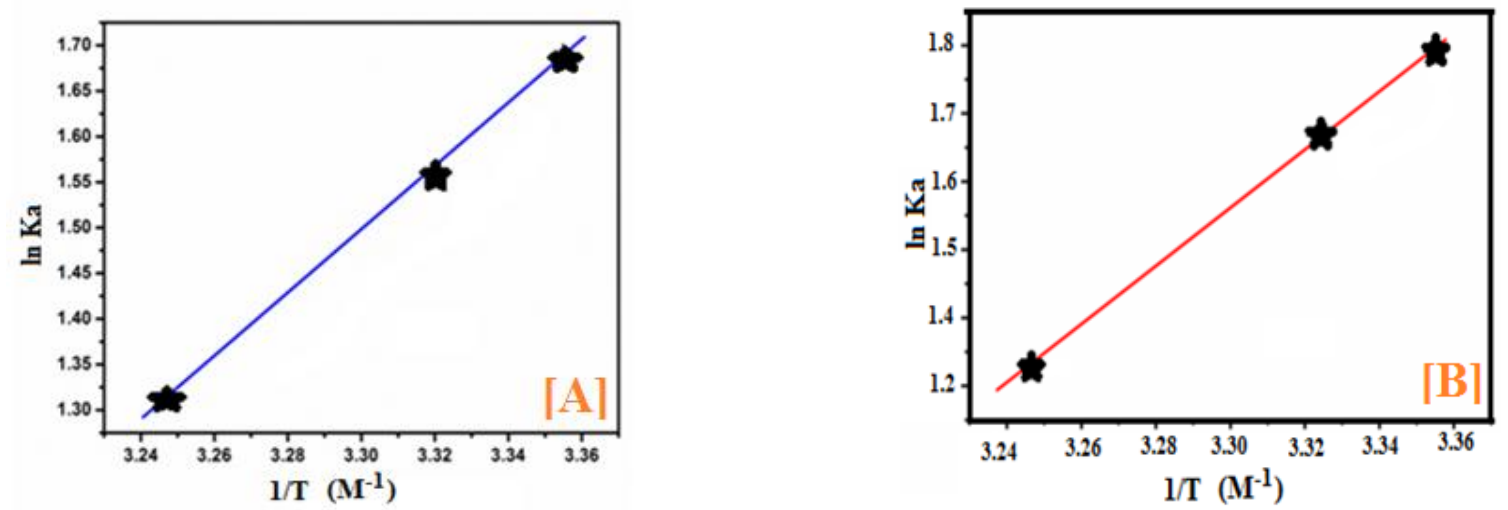

Fig. 5: Van't Hoff plot of $\operatorname{lnKa}$ Against $1 / \mathrm{T}\left(\mathrm{M}^{-1}\right)$ i.e., (A) BSA-BmimOS and (B) HSA-BmimOS.

Where, $\mathrm{K}_{\mathrm{a}}$ is comparable to the binding constant at the resultant $\mathrm{T}$, and $\mathrm{R}$ is the gas constant $(8.314 \mathrm{~J} / \mathrm{molK})$, correspondingly. Fig. 5 , viewing a good linear correlation between $\ln \mathrm{K}_{\mathrm{a}}$ vs $1 / \mathrm{T}$. As a result, $\Delta \mathrm{H}, \Delta \mathrm{S}$ and $\Delta \mathrm{G}$ were calculate according to the linear regression equation include in Fig.7. The calculate values $\Delta \mathrm{H}, \Delta \mathrm{S}$, and $\Delta \mathrm{G}$ were all shown Table 2 . The calculated negative value of $\Delta \mathrm{G}$ shows the binding process of ILs and HSA/BSA were spontaneous and negative values of $\Delta \mathrm{H}$ indicated that the binding process of ILs. HSA/BSA with IL complex was exothermic in nature. As a results suggest that higher the temperature is show the weaker interaction between [BmimOS]/ $\left[\mathrm{DmimBF}_{4}\right]$ with $\mathrm{HSA} / \mathrm{BSA}$.

Table 2: Thermodynamic Parameter of Complexation between BSA/HSA with [BmimOS]/ [DmimBF $\left.\mathrm{DF}_{4}\right]$

\begin{tabular}{|c|c|c|c|c|c|}
\hline Thermodynamic parameters & Temperature (K) & \multicolumn{2}{|c|}{ [BmimOS] } & \multicolumn{2}{|c|}{$\left[\mathrm{DmimBF}_{4}\right]$} \\
\hline \multirow{3}{*}{$\begin{array}{l}\Delta \mathrm{G} \\
\left(\mathrm{kJmol}^{-1}\right)\end{array}$} & 298 & -2.8 & -1.8 & -7.8 & -6.6 \\
\hline & 303 & -2.5 & -1.0 & -8.8 & -8.2 \\
\hline & 308 & -2.2 & -0.7 & -9.0 & -6.9 \\
\hline \multirow{3}{*}{$\begin{array}{l}\Delta \mathrm{H} \\
\left(\mathrm{kJmol}^{-1}\right)\end{array}$} & 298 & -6.31 & -6.46 & -5.47 & -9.02 \\
\hline & 303 & -4.62 & -3.53 & -3.65 & -4.69 \\
\hline & 308 & -2.72 & -2.17 & -3.39 & -7.96 \\
\hline \multirow{3}{*}{$\begin{array}{l}\Delta \mathrm{S} \\
\left(\mathrm{Jmol}^{-1} \mathrm{~K}^{-1}\right)\end{array}$} & 298 & 24.59 & 24.39 & 25.82 & 21.47 \\
\hline & 303 & 27.43 & 29.68 & 29.43 & 27.28 \\
\hline & 308 & 32.08 & 33.98 & 30.22 & 22.86 \\
\hline
\end{tabular}

\subsection{FT-IR spectrometry}

IR spectra of [BmimOS] (Fig. 6 A)/ [DmimBF 4$]$ (Fig. 7A) dispersed with HSA/BSA (Fig.6-8 B and 5B) were evidence using potassium bromide pellet in the scan range among $4000-400 \mathrm{~cm}^{-1}$. As a results obtained between the interaction of the HSA/BSA with [BmimOS]/ [DmimBF 4$]$ by FTIR is exposed in Figure 6-8. Globular proteins i.e., BSA/HSA shown two absorbing bands i.e., (i) amide first band $\mathrm{C}=\mathrm{O}$ str. at $1644 \mathrm{~cm}^{-1}$ and (ii)) amide second band C-N str. and $\mathrm{N}-\mathrm{H}$ bending mode at $1540 \mathrm{~cm}^{-1}$. IR spectra of amide first band is shifted from $1644 \mathrm{~cm}^{-1}$ to $1659 \mathrm{~cm}^{-1}$. Furthermore, amide II band shifted faintly from $1537 \mathrm{~cm}^{-1}$ to $1542 \mathrm{~cm}^{-1}$. These results convey that [BmimOS] also change the stretching vibration of $\mathrm{C}=\mathrm{O}$ groups in the protein polypeptides beside by the $\mathrm{C}-\mathrm{N}$ stretching and $\mathrm{N}-\mathrm{H}$ bending vibration modes. Usually, the aggregation of [BmimOS] with BSA/HSA resulted in the delay of the serum albumins $2^{0}$ structure and [BmimOS] on larger concentration explain greater persuade on the structure change of the serum albumins BSA/HSA which confirm well with the results experimental from fluorescence experiments.
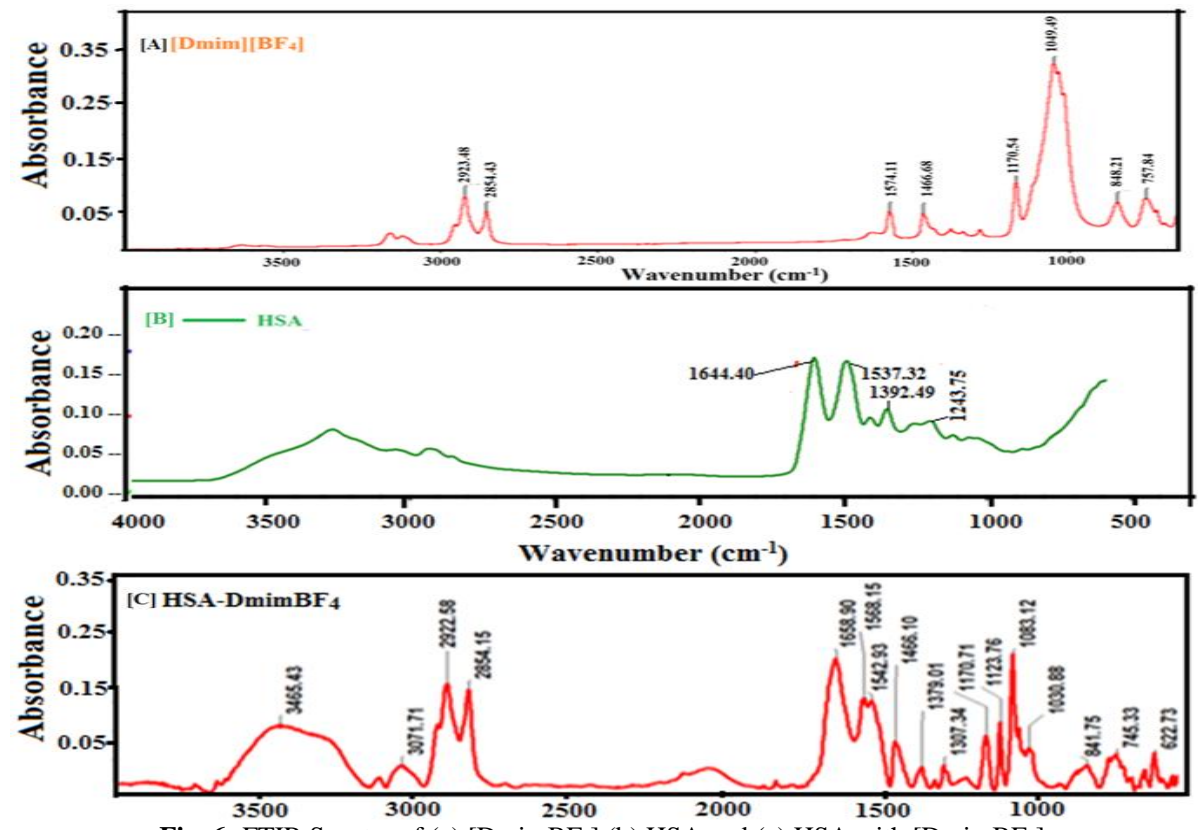

Fig. 6: FTIR Spectra of (a) [DmimBF 4 (b) HSA and (c) HSA with [DmimBF 4 ]. 

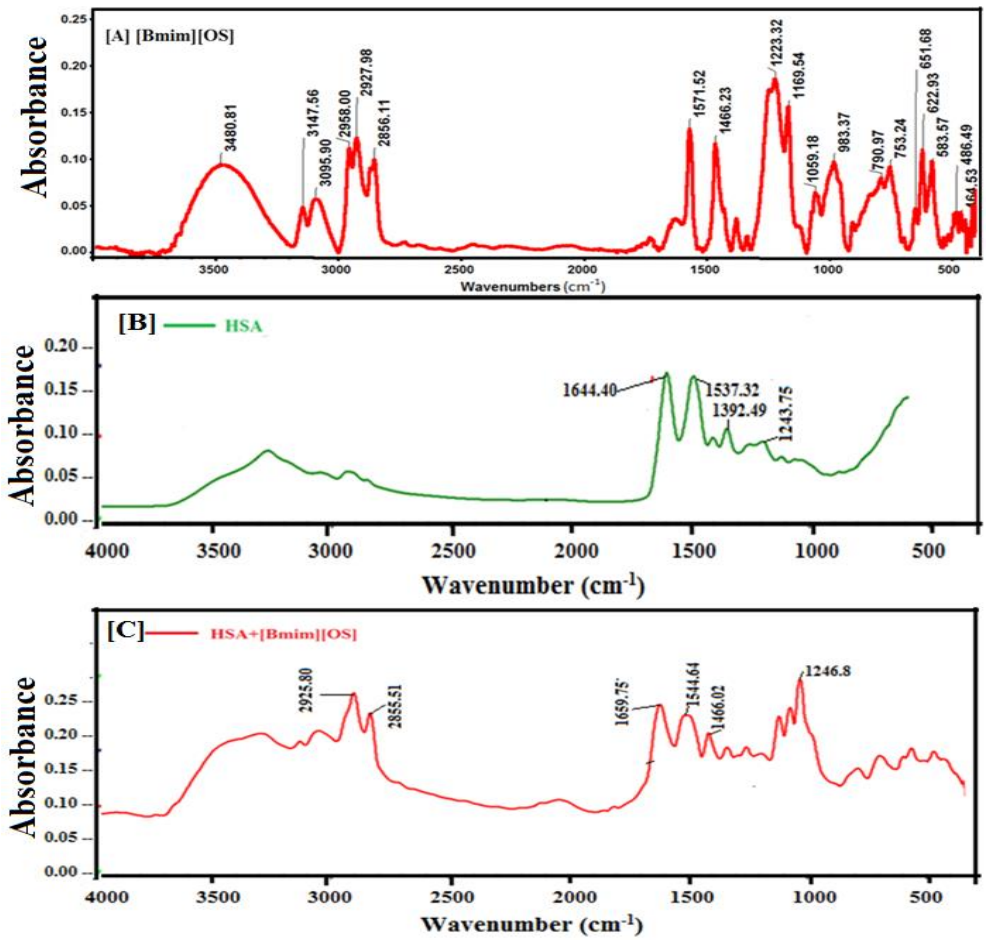

Fig. 7: FTIR Spectra of (a) [BmimOS] (b) HSA and (c) HSA with [BmimOS].
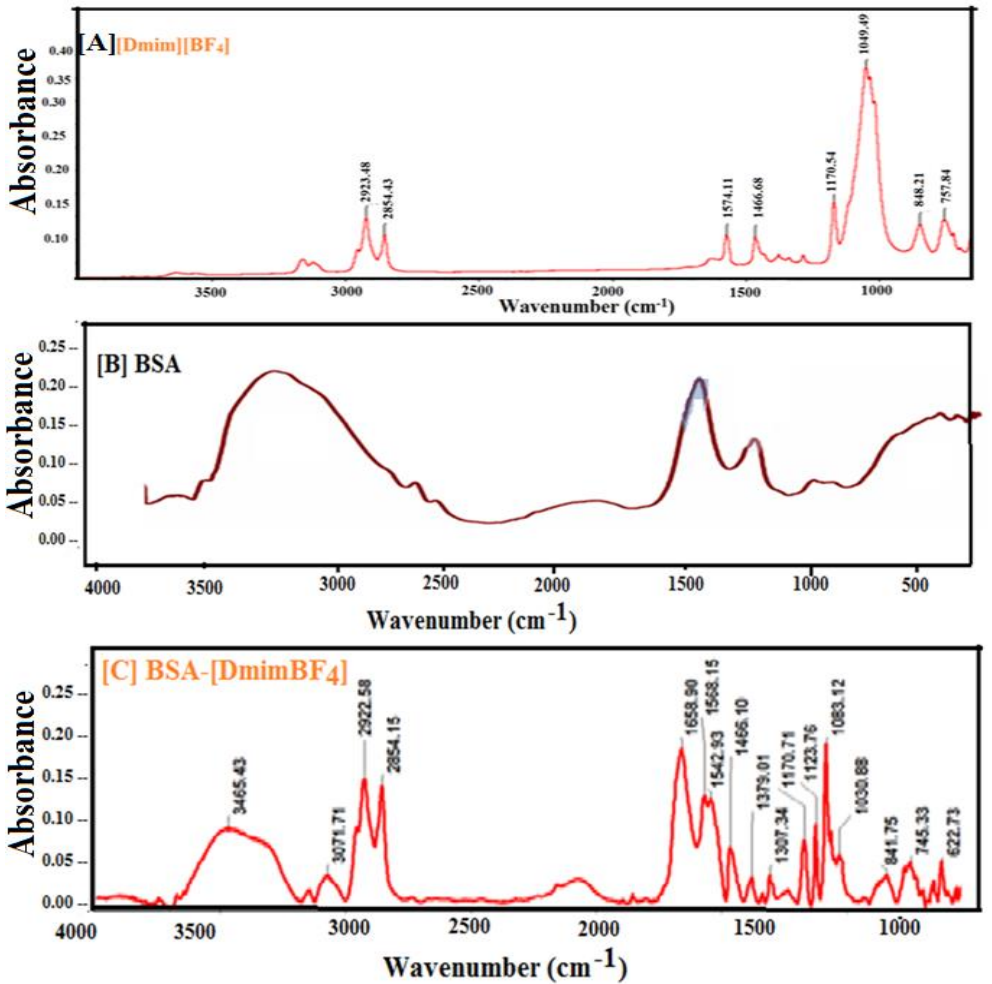

Fig. 8: FTIR Spectra Of (A) [Dmimbf 4 (B) BSA and (C) BSA With [Dmimbf 4 ].

Zhao et al. 2011, has been investigated the interaction between reserpine and bovine serum albumin (BSA) were studied using UV-vis absorption, fluorescence, and FTIR spectroscopy. The calculated binding constant $\mathrm{K}_{\mathrm{A}}$ of reserpine with BSA at three different temperatures 293,301 and $309 \mathrm{~K}$ was $1.63 \times 10^{5}, 1.78 \times 10^{5}$ and $2.35 \times 10^{5} \mathrm{moL}^{-1} \mathrm{~L}$ respectively. As results of fluorescence and FT-IR data show that the conformation of BSA was changed by the binding of reserpine.

\section{Conclusion}

Spectroscopic revealed that 1-butyl-3-methylimidazolium octylsulphate (BmimOS) and 1-decyl-3-methylimidazolium tetrafluoroborate $\left(\mathrm{DmimBF}_{4}\right)$ interact with HSA/BSA in the bulk earlier to interaction. The results explain that the fluorescence quenching of ILs with BSA/HSA go to static quenching. The fluorescence quenching decreased among the temperature rising. Thermodynamic parameters are responsible to the joining force between ILs and BSA/HSA was mostly hydrophobic interaction. Fluorescence expose the verify of HSA/BSA altered in the existence of $\left(\mathrm{DmimBF}_{4}\right) /(\mathrm{BmimOS})$. We have previously reported in our paper (Ghosh et al. 2018, Ghosh et al. 
2016) the interaction between serum albumin with nonmaterial's and surfactants from the description of conductivity, surface tension, fluorescence, UV-Vis, FT-IR and this study is an peace by our results.

\section{Acknowledgement}

All authors are thankful to Prof. M. K. Deb, SOS in Chemistry, Pt. Ravishankar Shukla University, Raipur (C.G.) for given FTIR instruments.

\section{Conflicts of interest}

Authors do not have any conflicts for this manuscript.

\section{References}

[1] Attri P, Venkatesu P \& Kumar A (2012) Water and a protic ionic liquid acted as refolding additives for chemically denatured enzymes, Org. biomol. Chem.,10, 7475-7478. https://doi.org/10.1039/c2ob26001h.

[2] Jha I, Attir P, \& Venkatesu P (2014) Unexpected effects of the alteration of structure and stability of myoglobin and hemoglobin in ammoniumbased ionic liquids, Phys. Chem. Phys., 162, 5514-5526. https://doi.org/10.1039/c3cp54398f.

[3] Sebastian W, Syguda A, Herrmann C, \& Weingartner H (2012) Steering the enzymatic activity of proteins by ionic liquids. A case study of the enzyme kinetics of yeast alcohol dehydrogenase, Phys. Chem. Chem. Phys., 14, 4635-4639. https://doi.org/10.1039/c2cp24041f.

[4] Zhao H, (2005) Effect of ions and other compatible solutes on enzyme activity, and its implication for biocatalysis using ionic liquids, J. Mol.Catal. B, 37, 16-25. https://doi.org/10.1016/j.molcatb.2005.08.007.

[5] Haberrler M, \& Steinhauser O (2011) On the influence of hydrated ionic liquids on the dynamical structure of model proteins: a computational study, Phys. Chem. Chem. Phys., 13, 17994-18004. https://doi.org/10.1039/c1cp22266j.

[6] Simon L M, Kotorman M, Garab G, \& Laczko I (2001) Structure and activity of $\alpha$-chymotrypsin and trypsin in aqueous organic media, Biochem. Biophys. Res. Comm., 280, 1367-1371. https://doi.org/10.1006/bbrc.2001.4282.

[7] Crevenna H, Rainer N N, Lamb D C, Soldner R W, \& Dzubiella J (2012) Effects of Hofmeister ions on the $\alpha$-helical structure of proteins, Biophys. J., 102, 907-915. https://doi.org/10.1016/j.bpj.2012.01.035.

[8] Ventura S P M, Santos L D F, Saraivab J A, \& Coutinho J A P (2012) Ionic liquids microemulsions: the key to Candida antarctica lipase B superactivity, Green Chem., 14, 1620-1625. https://doi.org/10.1039/c2gc35197h.

[9] Figueiredo A M, Sardinha J, Mooreb G R, \& Cabrita E J (2013) Protein destabilisation in ionic liquids: the role of preferential interactions in denaturation, Phys. Chem. Chem. Phys., 15, 19632-19643. https://doi.org/10.1039/c3cp53395f.

[10] Shao Q (2013) On the influence of hydrated imidazolium-based ionic liquid on protein structure stability: a molecular dynamics simulation study, J. Chem. Phys., 139, 115102-115108. https://doi.org/10.1063/1.4821588.

[11] Mahler J \& Perssion I (2012) A study of the hydration of the alkali metal ions in aqueous solution, Inorg. Chem., 51, 425-438. https://doi.org/10.1021/ic2018693.

[12] Pal A, \& Chaudhary S (2015) Thermodynamic and aggregation behavior of aqueous tetradecyltrimethylammonium bromide in the presence of the hydrophobic ionic liquid 3-methyl-1-pentylimidazolium hexafluorophosphate, J. Mol. Liq, 207, 67-72. https://doi.org/10.1016/j.molliq.2015.03.021.

[13] Chai J, Zhang H, Liu N, Liu N, Chai H, \& Liu Z (2015) Comparison between phase behavior of gemini imidazoliums and monomeric ionic liquid surfactants in W/O microemulsion systems, J. Dispersion Sci. Technol, 36, 129-135. https://doi.org/10.1080/01932691.2014.890108.

[14] Rouha M, \& Cummings P T (2015) Thickness-dependent structural arrangement in nano-confined imidazolium-based ionic liquid films, Phys. Chem. Chem. Phys, 17, 4152- 4159. https://doi.org/10.1039/C4CP05138F.

[15] Bernardes C E S, Shimizu K, Lopes J N C, Marquetand P, Heid E, Steinhauserc O, \& Schroder C (2016) Additive polarizabilities in ionic liquids, Phys. Chem. Chem. Phys., 18, $1665-1670$. https://doi.org/10.1039/C5CP06595J.

[16] Ghani N A, Yusoff R, Sairi N A, Aroua M K, \& Alias Y (2014) Density, Surface Tension, and Viscosity of Ionic Liquids (1-Ethyl-3methylimidazolium diethylphosphate and 1,3-Dimethylimidazolium dimethylphosphate) Aqueous Ternary Mixtures with MDEA, J. Chem. Eng. Data, 59, 1737-1746. https://doi.org/10.1021/je400562z.

[17] Saha S, Ray T, Basak S, \& Roy M N (2016) NMR, surface tension and conductivity studies to determine the inclusion mechanism: thermodynamics of host-guest inclusion complexes of natural amino acids in aqueous cyclodextrins, New J. Chem., 40, 651-661. https://doi.org/10.1039/C5NJ02179K.

[18] Singh T, Bharmoria P, Morikawa M, Kimizuka N, \& Kumar A (2012) Ionic liquids induced structural changes of bovine serum albumin in aqueous media: a detailed physicochemical and spectroscopic study, J. Phys. Chem. B, 116, 11924-11935. https://doi.org/10.1021/jp303609h.

[19] Patel R, Kumari M, Dohare N, Khan A B, Singh P, Malik M A, \& Kumar A (2016) Interaction between pyrrolidinium based ionic liquid and bovine serum albumin: a spectroscopic and molecular docking insight, Biochem Biochem., 5, 1-8. https://doi.org/10.4172/2161-1009.1000265.

[20] Kumari M, Singh UK, Singh P, \& Patel R (2017) Effect of n-butyl-n- methyl-morpholinium bromide ionic liquid on the conformation stability of human serum albumin, Chem. Select, 3, 1241-1249. https://doi.org/10.1002/slct.201601477.

[21] Reshma, Vaishnav S K, Karbhal I, Satnami M L, \& Ghosh K K (2018) Spectroscopic studies on in vitro molecular interaction of highly fluorescent carbon dots with different serum albumins, J. Mol. Liq.,255, 279-287. https://doi.org/10.1016/j.molliq.2018.01.146.

[22] Sinha S, Tikariha D, Lakra J, Yadav T, Kumari S, Saha S K, \& Ghosh K K (2016) Interaction of bovine serum albumin with cationic monomeric and dimeric surfactants: A comparative study, J. Mol. Liq., 218, 421-428. https://doi.org/10.1016/j.molliq.2016.02.052.

[23] Amar B T, Ghisaidoobe, \& Chung S J (2014) Intrinsic tryptophan fluorescence in the detection and analysis of proteins: a focus on förster resonance energy transfer techniques, Int. J. Mol. Sci., 12, 22518-22538. https://doi.org/10.3390/ijms151222518.

[24] Huang S, Qiu H, Xie J, Huang C, Su W, Hu B, \& Xiao Q (2016) Systematical investigation of in vitro molecular interaction between fluorescent carbon dots and human serum albumin, RSC Adv., 6, 44531-44542. https://doi.org/10.1039/C6RA01386D.

[25] Jana P, Patel N, Mukherjee T, Soppina V, \& Kanvah S (2019) A "turn-on" Michler's ketone-benzimidazole fluorescent probe for selective detection of serum albumins, New J. Chem., 43, 10859-10867. https://doi.org/10.1039/C9NJ01972C.

[26] Toprak M, Aydın B M, Arik M, \& Onganer Y (2011) Fluorescence quenching of fluorescein by Merocyanine 540 in liposomes, J. Luminescence, 11, 2286-2289. https://doi.org/10.1016/j.jlumin.2011.05.049.

[27] Carbery W P, Pacheco B P, Buccella D \& Turner D B (2019) Resolving the fluorescence quenching mechanism of an oxazine dye using ultrabroadband two-dimensional electronic spectroscopy, J. Phys. Chem. A, 123, 5072-5080 https://doi.org/10.1021/acs.jpca.9b03632.

[28] Zuo, Y Q, Yi, Zhong-Sheng, X, Jie, R, Yue-Fan, W, Yu-Cheng, L, \& Hong-Yan (2019) Molecular simulation and spectroscopic studies on the interaction between perfluorohexadecanoic acid and human serum albumin. Indian J. Biochem Bio., 56, 185-192.

[29] Kamal, J K A, \& Behere, D V (2019) Binding of heme to human serum albumin: Steady-state fluorescence, circular dichroism and optical difference spectroscopic studies. Indian J Biochem Bio, 42, 7-12. 
[30] Mathavan, V M K, Boh, B K, \& Tayyab, S (2009) Characterization of erythrosine B binding to bovine serum albumin and bilirubin displacement. Indian J Biochem Bio, 46, 325-331.

[31] Kamat, B P, Seetharamappa, J, \& Melwanki, M B (2004) Spectroscopic studies on the interaction of riboflavin with bovine serum albumin. Indian J Biochem Bio, 41, 173-178.

[32] Wang, T, Zhao, Z, Hua, J, \& Zhang, J (2011) Characterization of the interaction between reserpine and bovine serum albumin: Spectroscopic approaches. Indian J Biochem Bio, 48, 388-394. 